\title{
Non-contiguous finished genome sequence of the opportunistic oral pathogen Prevotella multisaccharivorax type strain (PPPA20 ${ }^{\mathrm{T}}$ )
}

\author{
Amrita Pati ${ }^{1}$, Sabine Gronow ${ }^{2}$, Megan Lu ${ }^{1,3}$, Alla Lapidus ${ }^{1}$, Matt Nolan ${ }^{1}$, Susan Lucas ${ }^{1}$, Nancy \\ Hammon ${ }^{1}$, Shweta Deshpande ${ }^{1}$, Jan-Fang Cheng ${ }^{1}$, Roxanne Tapia ${ }^{1,3}$, Cliff Han ${ }^{1,3}$, Lynne \\ Goodwin $^{1,3}$ Sam Pitluck ${ }^{1}$, Konstantinos Liolios ${ }^{1}$, Ioanna Pagani ${ }^{1}$, Konstantinos Mavromatis ${ }^{1}$, \\ Natalia Mikhailova' ${ }^{1}$ Marcel Huntemann' ${ }^{1}$, Amy Chen ${ }^{4}$, Krishna Palaniappan ${ }^{4}$, Miriam \\ Land $^{1,5}$, Loren Hauser ${ }^{1,5}$, John C. Detter ${ }^{1,3}$, Evelyne-Marie Brambilla ${ }^{2}$, Manfred Rohde ${ }^{6}$, \\ Markus Göker ${ }^{2}$, Tanja Woyke ${ }^{1}$, James Bristow ${ }^{1}$, Jonathan A. Eisen ${ }^{1,7}$, Victor Markowitz ${ }^{4}$, \\ Philip Hugenholtz ${ }^{1,8}$, Nikos C. Kyrpides ${ }^{1}$, Hans-Peter Klenk ${ }^{2 *}$, and Natalia Ivanova ${ }^{1}$ \\ ${ }^{1}$ DOE Joint Genome Institute, Walnut Creek, California, USA \\ 2 DSMZ - German Collection of Microorganisms and Cell Cultures GmbH, Braunschweig, \\ Germany \\ ${ }^{3}$ Los Alamos National Laboratory, Bioscience Division, Los Alamos, New Mexico, USA \\ ${ }^{4}$ Biological Data Management and Technology Center, Lawrence Berkeley National \\ Laboratory, Berkeley, California, USA \\ ${ }^{5}$ Oak Ridge National Laboratory, Oak Ridge, Tennessee, USA \\ ${ }^{6} \mathrm{HZI}$ - Helmholtz Centre for Infection Research, Braunschweig, Germany \\ ${ }^{7}$ University of California Davis Genome Center, Davis, California, USA \\ ${ }^{8}$ Australian Centre for Ecogenomics, School of Chemistry and Molecular Biosciences, The \\ University of Queensland, Brisbane, Australia \\ *Corresponding author: Hans-Peter Klenk
}

Keywords: obligately anaerobic, non-motile, Gram-negative, mesophilic, chemoorganotrophic, opportunistic pathogen, Prevotellaceae, GEBA

\begin{abstract}
Prevotella multisaccharivorax Sakamoto et al. 2005 is a species of the large genus Prevotella, which belongs to the family Prevotellaceae. The species is of medical interest because its members are able to cause diseases in the human oral cavity such as periodontitis, root caries and others. Although 77 Prevotella genomes have already been sequenced or are targeted for sequencing, this is only the second completed genome sequence of a type strain of a species within the genus Prevotella to be published. The 3,388,644 bp long genome is assembled in three non-contiguous contigs, harbors 2,876 protein-coding and 75 RNA genes and is a part of the Genomic Encyclopedia of Bacteria and Archaea project.
\end{abstract}

\section{Introduction}

Strain PPPA20T (= DSM $17128=$ JCM 12954) is the type strain of Prevotella multisaccharivorax [1]. Currently, there are about 50 species placed in the genus Prevotella [1]. The species epithet is derived from the Latin adjective multus meaning 'many/much', the Latin noun saccharum meaning 'sugar' and the Latin adjective vorax meaning 'liking to eat' referring to the metabolic properties of the species to digest a variety of carbohydrates [2]. $P$. multisaccharivorax strain PPPA20 ${ }^{\mathrm{T}}$ is considered to be an opportunistic pathogen and was isolated from subgingival plaque from a patient with chronic periodontitis. Additionally, five more strains isolated from the human oral cavity were placed in the species $P$. multisaccharivorax [2]. Using non-culture techniques on sites affected by endodontic and periodontal diseases, a large number of sequences have been found that belong to Prevotella and Prevotella-like bacteria. Many of those species have never been isolated or described [3]. The complex microbial community living in the rich ecological niche of the human oral cavity and its interaction with consumed food will be of lasting interest for medical and ecological reasons [4,5]. Here we present a summary classification and a set of features for P. multisaccharivorax PPPA20 ${ }^{\mathrm{T}}$, together with the description of the non-contiguous finished genomic sequencing and annotation. 


\section{Classification and features}

A representative genomic 16S rRNA sequence of $P$. multisaccharivorax PPPA20 ${ }^{\mathrm{T}}$ was compared using NCBI BLAST [6] under default settings (e.g., considering only the high-scoring segment pairs (HSPs) from the best 250 hits) with the most recent release of the Greengenes database [7] and the relative frequencies of taxa and keywords (reduced to their stem [8]) were determined, weighted by BLAST scores. The most frequently occurring genus was Prevotella (100.0\%) (14 hits in total). Regarding the single hit to sequences from members of the species, the average identity within HSPs was $100.0 \%$, whereas the average coverage by HSPs was $98.0 \%$. Regarding the nine hits to sequences from other members of the genus, the average identity within HSPs was $90.3 \%$, whereas the average coverage by HSPs was $66.5 \%$. Among all other species, the one yielding the highest score was Prevotella ruminicola (AF218618), which corresponded to an identity of 91.5\% and an HSP coverage of $66.3 \%$. (Note that the Greengenes database uses the INSDC (= EMBL/NCBI/DDBJ) annotation, which is not an authoritative source for nomenclature or classification.) The highest-scoring environmental sequence was AY550995 ('human carious dentine clone IDRCEC-0032'), which showed an identity of $99.8 \%$ and an HSP coverage of $94.5 \%$. The most frequently occurring keywords within the labels of environmental samples which yielded hits were 'fecal' (4.4\%), 'beef, cattl' (4.1\%), 'anim, coli, escherichia, feedlot, habitat, marc, pen, primari, secondari, stec, synecolog' (4.0\%), 'neg' (2.5\%) and 'fece' (2.4\%) (236 hits in total). The most frequently occurring keywords within the labels of environmental samples which yielded hits of a higher score than the highest scoring species were 'fece' (7.9\%), 'goeldi, marmoset' (4.8\%), 'microbiom' (4.3\%), 'aspect, canal, oral, root' (3.9\%) and 'rumen' (3.8\%) (54 hits in total). While some of these keywords correspond to the well known habitat of $P$. multisaccharivorax, others indicate additional habitats related to animals.

Figure 1 shows the phylogenetic neighborhood of $P$. multisaccharivorax in a $16 \mathrm{~S}$ rRNA based tree. The sequences of the four 16S rRNA gene copies in the genome differ from each other by up to two nucleotides, and differ by up to two nucleotides from the previously published 16S rRNA sequence AB200414.

The cells of $P$. multisaccharivorax generally have the shape of rods $(0.8 \times 2.5-8.3 \mu \mathrm{m})$ and occur singly or in pairs (Figure 2). They can also form longer filaments. P. multisaccharivorax is a Gram-negative, non spore-forming bacterium (Table 1). The organism is described as non-motile and only four genes associated with motility were identified in the genome (see below). P. multisaccharivorax grows well at $37^{\circ} \mathrm{C}$, is strictly anaerobic, chemoorganotrophic and is able to ferment cellobiose, glucose, glycerol, lactose, maltose, mannose, melezitose, raffinose, rhamnose, sorbitol, sucrose, trehalose and xylose [2]. Acid production from arabinose and salicin is variable. The organism does not reduce nitrate or produce indole from tryptophan but it hydrolyzes esculin and digests gelatin [2]. Growth of P. multisaccharivorax is inhibited by the addition of $20 \%$ bile. Major fermentation products are succinic and acetic acid, isovaleric acid is produced in small amounts [2]. Activities of glucose-6-phosphate dehydrogenase (G6PDH) and 6-phosphogluconate dehydrogenase (6GPDH) were not detected in isolates of this species, whereas malate dehydrogenase and glutamate dehydrogenase activities were detected in all strains. P. multisaccharivorax produces acid and alkaline phosphatase, $\beta$-galactosidase, $\alpha$ and $\beta$-glucosidase, $N$-acetyl- $\beta$-glucosaminidase, $\alpha$ aminofuranosidase and alanine aminopeptidase. The organism has no demonstrable esterase (C4), esterase lipase (C4), lipase (C4), leucine arylamidase, valine arylamidase, cystine arylamidase, pyroglutamic acid arylamidase, trypsin, chymotrypsin, $\beta$-glucuronidase, $\alpha$-mannosidase, $\alpha$-fucosidase, arginine aminopeptidase, leucine aminopeptidase, proline aminopeptidase, tyrosine aminopeptidase, phenylalanine aminopeptidase, urease or catalase activity [2].

\section{Chemotaxonomy}

In contrast to other Prevotella species all strains of $P$. multisaccharivorax harbor the menaquinones MK-12 (40-55\%) and MK-13 (40-45\%) in large amounts, whereas MK-10 (1-3\%) and MK-11 (8$10 \%)$ were found only in small amounts [2]. The fatty acid pattern for all strains of $P$. multisaccharivorax revealed $\mathrm{C}_{18: 1} \omega 9 \mathrm{c}(21.7 \%)$ and $\mathrm{C}_{16: 0}(12.9 \%)$ as major compounds as well as iso- $\mathrm{C}_{17: 0} 3-0 \mathrm{H}(9.2 \%)$, anteiso- $\mathrm{C}_{15: 0}(7.8 \%), \mathrm{C}_{18: 2} \omega 6,9 \mathrm{c}(7.5 \%)$ and iso- $\mathrm{C}_{15: 0}$ $(6.4 \%)$ in smaller amounts [2]. Additionally, the unusual dimethyl acetals were found with $\mathrm{C}_{16: 0}$ dimethyl aldehyde in the highest amount of $8.2 \%$. This clearly distinguishes the species of $P$. multisaccharivorax from other related Prevotella species [2]. 


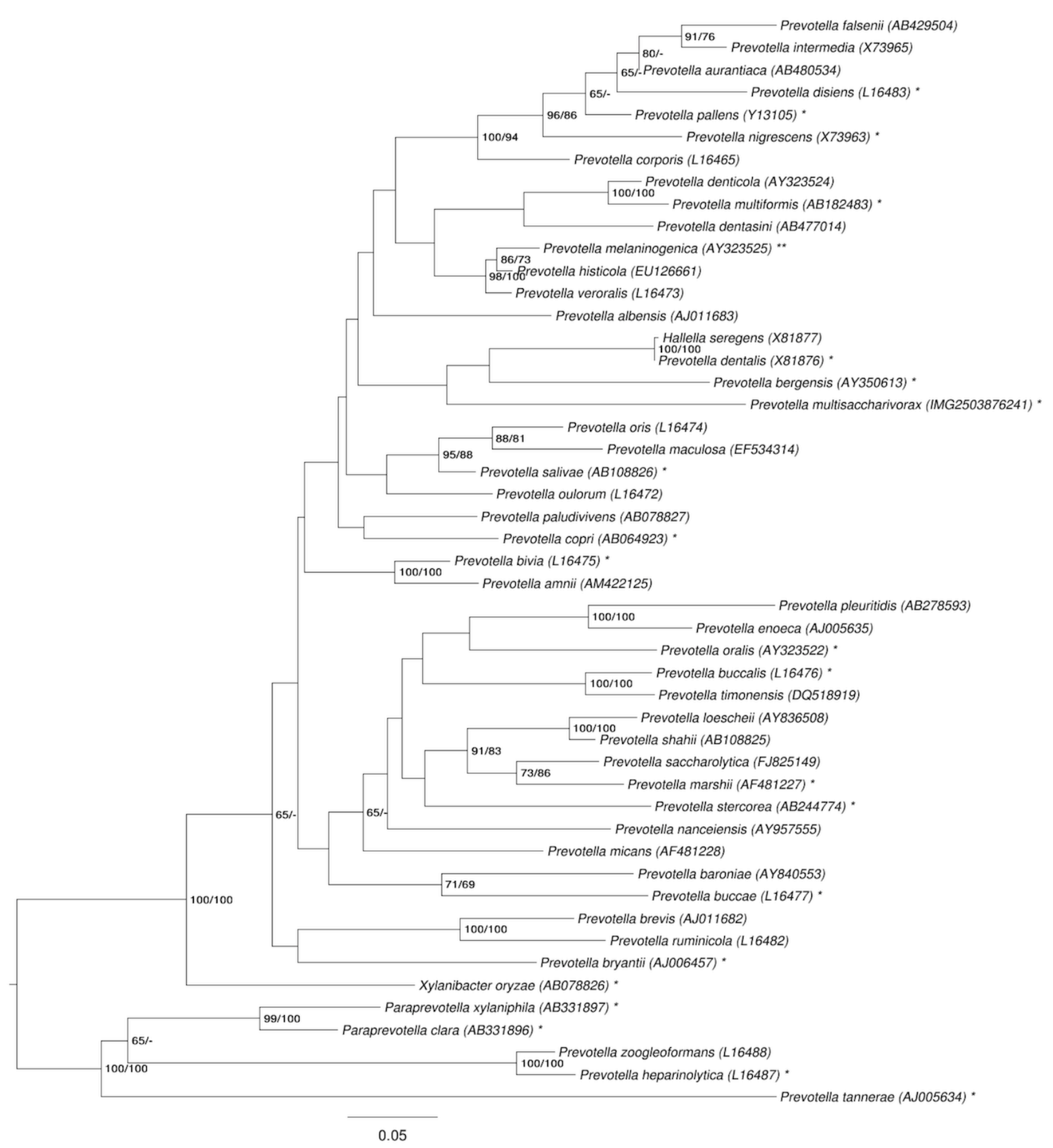

Figure 1. Phylogenetic tree highlighting the position of $P$. multisaccharivorax relative to the type strains of the other species within the family. The tree was inferred from 1,425 aligned characters $[9,10]$ of the $16 \mathrm{~S}$ rRNA gene sequence under the maximum likelihood (ML) criterion [11]. Rooting was done initially using the midpoint method [12] and then checked for its agreement with the current classification (Table 1). The branches are scaled in terms of the expected number of substitutions per site. Numbers adjacent to the branches are support values from 600 ML bootstrap replicates [13] (left) and from 1,000 maximum parsimony bootstrap replicates [14] (right) if larger than $60 \%$. Lineages with type strain genome sequencing projects registered in GOLD [15] are labeled with one asterisk, those also listed as 'Complete and Published' should be labeled with two asterisks: P. ruminicola [16] and P. melaninogenica (CP002122/CP002123) 


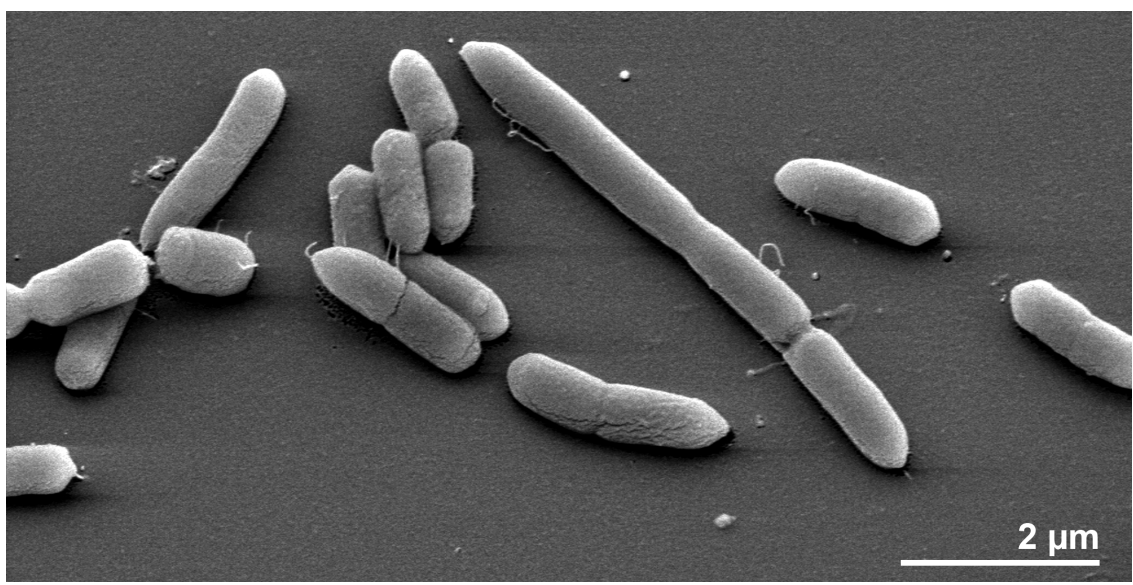

Figure 2. Scanning electron micrograph of P. multisaccharivorax PPPA20 ${ }^{\top}$

Table 1. Classification and general features of $P$. multisaccharivorax PPPA2 $20^{\top}$ according the MIGS recommendations [17] and the NamesforLife database [1].

\begin{tabular}{|c|c|c|c|}
\hline MIGS ID & Property & Term & Evidence code \\
\hline & \multirow{8}{*}{ Current classification } & Domain Bacteria & TAS [18] \\
\hline & & Phylum "Bacteroidetes" & TAS [19] \\
\hline & & Class "Bacteroidia" & TAS [20] \\
\hline & & Order "Bacteroidales" & TAS [21] \\
\hline & & Family "Prevotellaceae" & TAS [21] \\
\hline & & Genus Prevotella & TAS $[22,23]$ \\
\hline & & Species Prevotella multisaccharivorax & TAS [2] \\
\hline & & Type strain PPPA20 & TAS [2] \\
\hline & Gram stain & negative & TAS [2] \\
\hline & Cell shape & rod-shaped & TAS [2] \\
\hline & Motility & non-motile & TAS [2] \\
\hline & Sporulation & none & TAS [2] \\
\hline & Temperature range & mesophilic & TAS [2] \\
\hline & Optimum temperature & $37^{\circ} \mathrm{C}$ & TAS [2] \\
\hline & Salinity & physiological & TAS [2] \\
\hline \multirow[t]{3}{*}{ MIGS-22 } & Oxygen requirement & obligately anaerobic & TAS [2] \\
\hline & Carbon source & carbohydrates & TAS [2] \\
\hline & Energy metabolism & chemoorganotrophic & TAS [2] \\
\hline MIGS-6 & Habitat & host, human oral microflora & TAS [2] \\
\hline MIGS-15 & Biotic relationship & free-living & NAS \\
\hline \multirow[t]{3}{*}{ MIGS-14 } & Pathogenicity & opportunistic pathogen & TAS [2] \\
\hline & Biosafety level & 2 & TAS [24] \\
\hline & Isolation & subgingival plaque, chronic periodontitis & TAS [2] \\
\hline MIGS-4 & Geographic location & Japan & TAS [2] \\
\hline MIGS-5 & Sample collection time & December 9, 2002 & IDA \\
\hline MIGS-4.1 & Latitude & not reported & \\
\hline MIGS-4.2 & Longitude & not reported & \\
\hline MIGS-4.3 & Depth & not reported & \\
\hline MIGS-4.4 & Altitude & not reported & \\
\hline
\end{tabular}

Evidence codes - IDA: Inferred from Direct Assay (first time in publication); TAS: Traceable Author Statement (i.e., a direct report exists in the literature); NAS: Non-traceable Author Statement (i.e., not directly observed for the living, isolated sample, but based on a generally accepted property for the species, or anecdotal evidence). These evidence codes are from of the Gene Ontology project [25]. If the evidence code is IDA, the property was directly observed by one of the authors or an expert mentioned in the acknowledgements. 


\section{Genome sequencing and annotation Genome project history}

This organism was selected for sequencing on the basis of its phylogenetic position [26], and is part of the Genomic Encyclopedia of Bacteria and Archaea project [27]. The genome project is deposited in the Genomes On Line Database [15] and the complete genome sequence is deposited in GenBank. Sequencing, finishing and annotation were performed by the DOE Joint Genome Institute (JGI). A summary of the project information is shown in Table 2.

Table 2. Genome sequencing project information

\begin{tabular}{|c|c|c|}
\hline MIGS ID & Property & Term \\
\hline MIGS-31 & Finishing quality & Non-contiguous finished \\
\hline MIGS-28 & Libraries used & $\begin{array}{l}\text { Three genomic libraries: one } 454 \text { pyrosequence standard library, } \\
\text { one } 454 \text { PE library (10 kb insert size), one Illumina library }\end{array}$ \\
\hline MIGS-29 & Sequencing platforms & Illumina GAii, 454 GS FLX Titanium \\
\hline MIGS-31.2 & Sequencing coverage & $290.0 \times$ Illumina; $48.0 \times$ pyrosequence \\
\hline MIGS-30 & Assemblers & Newbler version 2.3, Velvet 0.7.63, phrap SPS 4.24 \\
\hline \multirow[t]{6}{*}{ MIGS-32 } & Gene calling method & Prodigal 1.4, GenePRIMP \\
\hline & INSDC ID & AFJE00000000 GL945015-GL945017 \\
\hline & Genbank Date of Release & June 20,2011 \\
\hline & GOLD ID & Gi05358 \\
\hline & NCBI project ID & 41513 \\
\hline & Database: IMG-GEBA & 2503754046 \\
\hline \multirow[t]{2}{*}{ MIGS-13 } & Source material identifier & DSM 17128 \\
\hline & Project relevance & Tree of Life, GEBA \\
\hline
\end{tabular}

\section{Growth conditions and DNA isolation}

P. multisaccharivorax PPPA20T, DSM 17128, was grown anaerobically in DSMZ medium 104 (PYGmedium) [28] at $37^{\circ} \mathrm{C}$. DNA was isolated from 0.5$1 \mathrm{~g}$ of cell paste using MasterPure Gram-positive DNA purification kit (Epicentre MGP04100) following the standard protocol as recommended by the manufacturer with modification st/DL for cell lysis as described in Wu et al. 2009 [27]. DNA is available through the DNA Bank Network [29].

\section{Genome sequencing and assembly}

The genome was sequenced using a combination of Illumina and 454 sequencing platforms. All general aspects of library construction and sequencing can be found at the JGI website [30]. Pyrosequencing reads were assembled using the Newbler assembler (Roche). The initial Newbler assembly consisting of 154 contigs in five scaffolds was converted into a phrap [31] assembly by making fake reads from the consensus, to collect the read pairs in the 454 paired end library. Illumina GAii sequencing data $(1,043.6 \mathrm{Mb})$ was assembled with Velvet [32] and the consensus sequences were shredded into $2.0 \mathrm{~kb}$ overlapped fake reads and assembled together with the 454 data. The 454 draft assembly was based on $135.4 \mathrm{Mb} 454$ standard data and all of the 454 paired end data. Newbler parameters are -consed -a 50 -l 350 -g -m -ml 20. The Phred/Phrap/Consed software package [31] was used for sequence assembly and quality assessment in the subsequent finishing process. After the shotgun stage, reads were assembled with parallel phrap (High Performance Software, LLC). Possible mis-assemblies were corrected with gapResolution [30], Dupfinisher [33], or sequencing cloned bridging PCR fragments with subcloning or transposon bombing (Epicentre Biotechnologies, Madison, WI). Gaps between contigs were closed by editing in Consed, by PCR and by Bubble PCR primer walks (J.-F. Chang, unpublished). A total of 218 additional reactions were necessary to close gaps and to raise the quality of the finished sequence. Illumina reads were also used to correct potential base errors and increase consensus quality using a software Polisher developed at JGI [34].

The error rate of the completed genome sequence is less than 1 in 100,000. Together, the combination of the Illumina and 454 sequencing platforms provided $338 \times$ coverage of the genome. The final assembly contained 325,939 pyrosequence and $28,989,384$ Illumina reads. 


\section{Genome annotation}

Genes were identified using Prodigal [35] as part of the Oak Ridge National Laboratory genome annotation pipeline, followed by a round of manual curation using the JGI GenePRIMP pipeline [36]. The predicted CDSs were translated and used to search the National Center for Biotechnology Information (NCBI) non-redundant database, UniProt, TIGR-Fam, Pfam, PRIAM, KEGG, COG, and InterPro databases. Additional gene prediction analysis and functional annotation was performed within the Integrated Microbial Genomes - Expert Review (IMG-ER) platform [37].

\section{Genome properties}

The assembled genome sequence consists of three non-contiguous contigs with a length of 3,334,154 bp, 47,474 bp and 7,016 bp with a $\mathrm{G}+\mathrm{C}$ content of $48.3 \%$ (Figure 3 and Table 3 ). Of the 2,951 genes predicted, 2,876 were protein-coding genes, and 75 RNAs; 166 pseudogenes were also identified. The majority of the protein-coding genes $(60.5 \%)$ were assigned with a putative function while the remaining ones were annotated as hypothetical proteins. The distribution of genes into COGs functional categories is presented in Table 4.

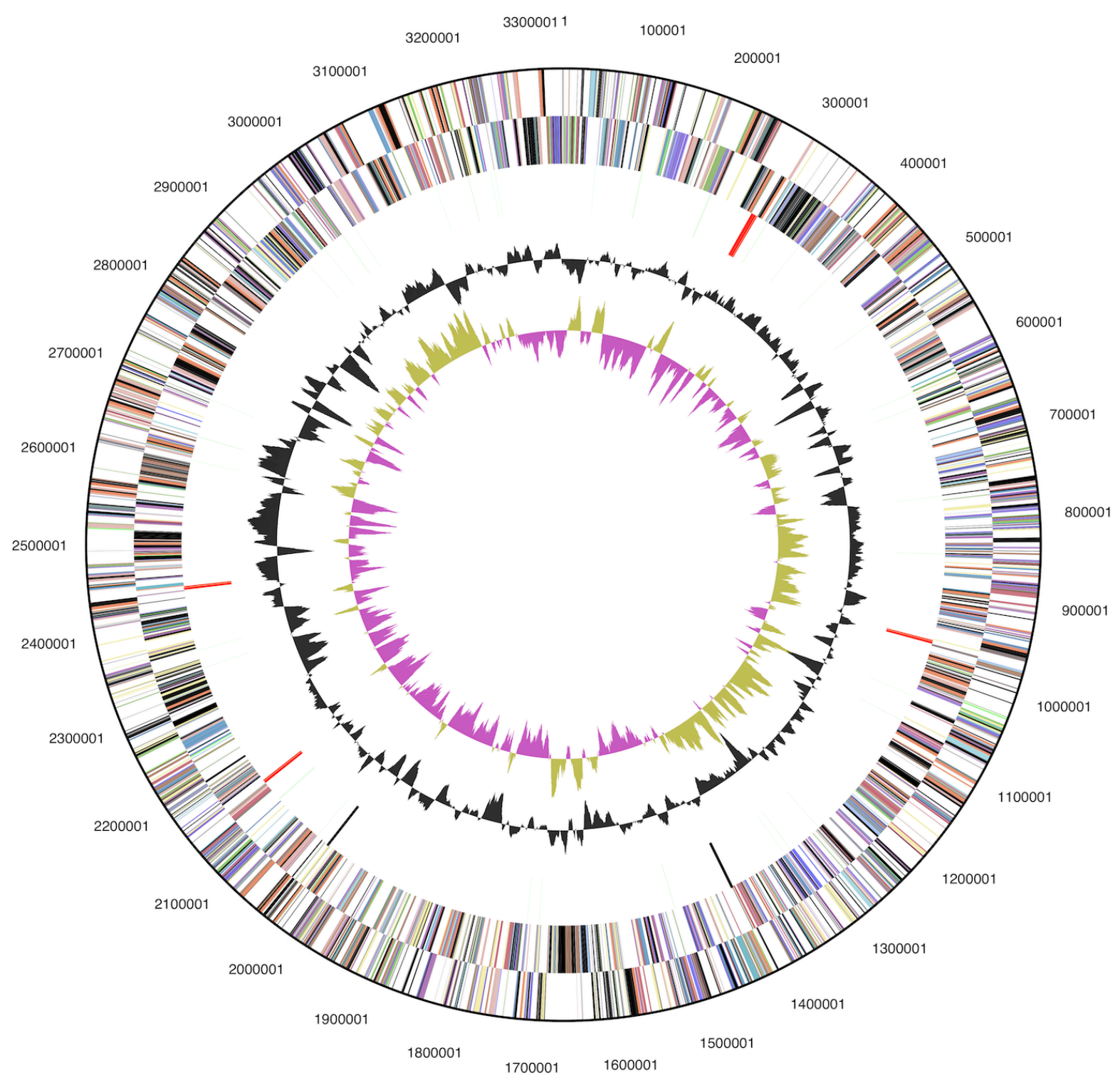

Figure 3. Graphical map of the largest scaffold. From outside to the center: Genes on forward strand (color by COG categories), Genes on reverse strand (color by COG categories), RNA genes (tRNAs green, rRNAs red, other RNAs black), GC content, GC skew. 
Table 3. Genome Statistics

\begin{tabular}{lrr}
\hline Attribute & Value & \% of Total \\
\hline Genome size (bp) & $3,388,644$ & $100.00 \%$ \\
DNA coding region (bp) & $2,970,483$ & $87.66 \%$ \\
DNA G+C content (bp) & $1,636,375$ & $48.31 \%$ \\
Number of scaffolds & 3 & \\
Total genes & 2,951 & $100.00 \%$ \\
RNA genes & 75 & $2.54 \%$ \\
rRNA operons & $4-6$ & \\
Protein-coding genes & 2,876 & $97.46 \%$ \\
Pseudo genes & 166 & $5.63 \%$ \\
Genes in paralog clusters & 438 & $14.84 \%$ \\
Genes assigned to COGs & 1,659 & $56.22 \%$ \\
Genes assigned Pfam domains & 1,864 & $63.17 \%$ \\
Genes with signal peptides & 782 & $26.50 \%$ \\
Genes with transmembrane helices & 588 & $19.93 \%$ \\
CRISPR repeats & 3 & \\
\hline
\end{tabular}

Table 4. Number of genes associated with the general COG functional categories

\begin{tabular}{crrl}
\hline Code & value & \%age & Description \\
\hline J & 138 & 7.7 & Translation, ribosomal structure and biogenesis \\
A & 0 & 0.0 & RNA processing and modification \\
K & 102 & 5.7 & Transcription \\
L & 183 & 10.1 & Replication, recombination and repair \\
B & 0 & 0.0 & Chromatin structure and dynamics \\
D & 26 & 1.4 & Cell cycle control, cell division, chromosome partitioning \\
Y & 0 & 0.0 & Nuclear structure \\
V & 46 & 2.6 & Defense mechanisms \\
T & 63 & 3.5 & Signal transduction mechanisms \\
M & 155 & 8.6 & Cell wall/membrane/envelope biogenesis \\
N & 4 & 0.2 & Cell motility \\
Z & 0 & 0.0 & Cytoskeleton \\
W & 0 & 0.0 & Extracellular structures \\
U & 31 & 1.7 & Intracellular trafficking, secretion, and vesicular transport \\
O & 69 & 3.8 & Posttranslational modification, protein turnover, chaperones \\
C & 90 & 5.0 & Energy production and conversion \\
G & 145 & 8.0 & Carbohydrate transport and metabolism \\
E & 132 & 7.3 & Amino acid transport and metabolism \\
F & 59 & 3.3 & Nucleotide transport and metabolism \\
H & 74 & 4.1 & Coenzyme transport and metabolism \\
I & 56 & 3.1 & Lipid transport and metabolism \\
P & 120 & 6.7 & Inorganic ion transport and metabolism \\
Q & 27 & 1.5 & Secondary metabolites biosynthesis, transport and catabolism \\
R & 202 & 11.2 & General function prediction only \\
S & 82 & 4.6 & Function unknown \\
- & 1,292 & 43.8 & Not in COGs \\
\hline & & &
\end{tabular}




\section{Acknowledgements}

We would like to gratefully acknowledge the help of Sabine Welnitz (DSMZ) for growing P. multisaccharivorax cultures. This work was performed under the auspices of the US Department of Energy Office of Science, Biological and Environmental Research Program, and by the University of California, Lawrence Berkeley National Laboratory under contract No. DE-AC02-

\section{References}

1. Garrity G. NamesforLife. BrowserTool takes expertise out of the database and puts it right in the browser. Microbiol Today 2010; 37:9.

2. Sakamoto M, Umeda M, Ishikawa I, Benno Y. Prevotella multisaccharivorax sp. nov., isolated from human subgingival plaque. Int / Syst Evol Microbiol 2005; 55:1839-1843. PubMed doi:10.1099/ijs.0.63739-0

3. Preza D, Olsen I, Aas JA, Willumsen T, Grinde B, Paster BJ. Bacterial profiles of root caries in elderly patients. J Clin Microbiol 2008; 46:2015-2021. PubMed doi:10.1128/JCM.02411-07

4. Rôças IN, Siqueira JF, Jr. Prevalence of new candidate pathogens Prevotella baroniae, Prevotella multisaccharivorax and as-yet-uncultivated Bacteroidetes clone X083 in primary endodontic infections. J Endod 2009; 35:1359-1362. PubMed doi:10.1016/j.joen.2009.05.033

5. Siqueira JF, Jr., Rôças IN. The oral microbiota: general overview, taxonomy, and nucleic acid techniques. Methods Mol Biol 2010; 666:55-69. PubMed doi:10.1007/978-1-60761-820-1_5

6. Altschul SF, Gish W, Miller W, Myers EW, Lipman DJ. Basic local alignment search tool. J Mol Biol 1990; 215:403-410. PubMed

7. DeSantis TZ, Hugenholtz $\mathrm{P}$, Larsen N, Rojas $\mathrm{M}$, Brodie EL, Keller K, Huber T, Dalevi D, Hu P, Andersen GL. Greengenes, a chimera-checked 16S rRNA gene database and workbench compatible with ARB. Appl Environ Microbiol 2006; 72:5069-5072. PubMed doi:10.1128/AEM.03006-05

8. Porter MF. An algorithm for suffix stripping. Program: electronic library and information systems 1980; 14:130-137.

9. Lee C, Grasso C, Sharlow MF. Multiple sequence alignment using partial order graphs. Bioinformatics 2002; 18:452-464. PubMed doi:10.1093/bioinformatics/18.3.452

10. Castresana J. Selection of conserved blocks from multiple alignments for their use in phylogenetic
05CH11231, Lawrence Livermore National Laboratory under Contract No. DE-AC52-07NA27344, and Los Alamos National Laboratory under contract No. DEAC02-06NA25396, UT-Battelle and Oak Ridge National Laboratory under contract DE-AC05-000R22725, as well as German Research Foundation (DFG) INST 599/1-2.

analysis. Mol Biol Evol 2000; 17:540-552. PubMed

11. Stamatakis A, Hoover P, Rougemont J. A rapid bootstrap algorithm for the RAxML web-servers. Syst Biol 2008; 57:758-771. PubMed doi:10.1080/10635150802429642

12. Hess PN, De Moraes Russo CA. An empirical test of the midpoint rooting method. Biol / Linn SoC Lond 2007; 92:669-674. doi:10.1111/j.10958312.2007.00864.x

13. Pattengale ND, Alipour $M$, Bininda-Emonds ORP, Moret BME, Stamatakis A. How many bootstrap replicates are necessary? Lect Notes Comput Sci 2009; 5541:184-200. doi:10.1007/978-3-642$\underline{02008-7 \quad 13}$

14. Swofford DL. PAUP*: Phylogenetic Analysis Using Parsimony (*and Other Methods), Version 4.0 b10. Sinauer Associates, Sunderland, 2002.

15. Liolios K, Chen IM, Mavromatis K, Tavernarakis $\mathrm{N}$, Hugenholtz P, Markowitz VM, Kyrpides NC. The Genomes OnLine Database (GOLD) in 2009: status of genomic and metagenomic projects and their associated metadata. Nucleic Acids Res 2010; 38:D346-D354. PubMed doi:10.1093/nar/gkp848

16. Purushe J, Foulds DE, Morrison M, White BA, Mackie RI. North American Consortium for Rumen Bacteria, Coultinho PM, Henrissat G, Nelson KE. Comparative genome anaylsis of Prevotella ruminicola and Prevotella bryantii: insight into their environmental niche. Microb Ecol 2010; 60:721-729. PubMed doi:10.1007/s00248-010$\underline{9692-8}$

17. Field D, Garrity G, Gray T, Morrison N, Selengut J, Sterk P, Tatusova T, Thomson N, Allen MJ, Angiuoli SV, et al. The minimum information about a genome sequence (MIGS) specification. Nat Biotechnol 2008; 26:541-547. PubMed doi:10.1038/nbt1360

18. Woese CR, Kandler O, Wheelis ML. Towards a natural system of organisms: proposal for the domains Archaea, Bacteria, and Eucarya. Proc Natl 
Acad Sci USA 1990; 87:4576-4579. PubMed doi:10.1073/pnas.87.12.4576

19. Garrity GM, Holt JG. The Road Map to the Manual. In: Garrity GM, Boone DR, Castenholz RW (eds), Bergey's Manual of Systematic Bacteriology, Second Edition, Volume 1, Springer, New York, 2001, p. 119-169.

20. Ludwig W, Euzeby J, Whitman WG. Draft taxonomic outline of the Bacteroidetes, Planctomycetes, Chlamydiae, Spirochaetes, Fibrobacteres, Fusobacteria, Acidobacteria, Verrucomicrobia, Dictyoglomi, and Gemmatimonadetes. http://www.bergeys.org/outlines/Bergeys Vol 4 Outline.pdf. Taxonomic Outline 2008.

21. Garrity GM, Holt JG. 2001. Taxonomic outline of the Archaea and Bacteria, p. 155-166. In: Garrity GM, Boone RR, Castenholz RW (eds), Bergey's Manual of Systematic Bacteriology, 2nd ed, vol. 1. Springer, New York.

22. Shah HN, Collins DM. Prevotella, a new genus to include Bacteroides melaninogenicus and related species formerly classified in the genus Bacteroides. Int J Syst Bacteriol 1990; 40:205-208. PubMed doi:10.1099/00207713-40-2-205

23. Willems A, Collins MD. 16S rRNA gene similarities indicate that Hallella seregens (Moore and Moore) and Mitsuokella dentalis (Haapasalo et al.) are genealogically highly related and are members of the genus Prevotella: emended description of the genus Prevotella (Shah and Collins) and description of Prevotella dentalis comb. nov. Int J Syst Bacteriol 1995; 45:832-836. PubMed doi:10.1099/00207713-45-4-832

24. BAuA. Classification of bacteria and archaea in risk groups. TRBA 2010; 466:173.

25. Ashburner M, Ball CA, Blake JA, Botstein D, Butler $\mathrm{H}$, Cherry JM, Davis AP, Dolinski K, Dwight SS, Eppig JT, et al. Gene Ontology: tool for the unification of biology. Nat Genet 2000; 25:25-29. $\underline{\text { PubMed doi:10.1038/75556 }}$

26. Klenk HP, Göker M. En route to a genome-based classification of Archaea and Bacteria? Syst Appl Microbiol 2010; 33:175-182. PubMed doi:10.1016/j.syapm.2010.03.003

27. Wu D, Hugenholtz P, Mavromatis K, Pukall R, Dalin E, Ivanova NN, Kunin V, Goodwin L, Wu $M$, Tindall BJ, et al. A phylogeny-driven genomic encyclopaedia of Bacteria and Archaea. Nature 2009; 462:1056-1060. PubMed doi:10.1038/nature08656

28. List of growth media used at DSMZ: http://www.dsmz.de/microorganisms/media_list.p hp.

29. Gemeinholzer B, Dröge G, Zetzsche H, Haszprunar G, Klenk HP, Güntsch A, Berendsohn WG, Wägele JW. The DNA Bank Network: the start from a German initiative. Biopreservation and Biobanking 2011; 9:51-55. doi:10.1089/bio.2010.0029

30. The DOE Joint Genome Institute. http://www.jgi.doe.gov

31. Phrap and Phred for Windows. MacOS, Linux, and Unix. http://www.phrap.com

32. Zerbino DR, Birney E. Velvet: algorithms for de novo short read assembly using de Bruijn graphs. Genome Res 2008; 18:821-829. PubMed doi:10.1101/gr.074492.107

33. Han C, Chain P. 2006. Finishing repeat regions automatically with Dupfinisher. In: Proceeding of the 2006 international conference on bioinformatics \& computational biology. Arabnia HR, Valafar H (eds), CSREA Press. June 26-29, 2006: $141-146$

34. Lapidus A, LaButti K, Foster B, Lowry S, Trong S, Goltsman E. POLISHER: An effective tool for using ultra short reads in microbial genome assembly and finishing. AGBT, Marco Island, FL, 2008.

35. Hyatt D, Chen GL, LoCascio PF, Land ML, Larimer FW, Hauser LJ. Prodigal: prokaryotic gene recognition and translation initiation site identification. BMC Bioinformatics 2010; 11:119. PubMed doi:10.1186/1471-2105-11-119

36. Pati A, Ivanova NN, Mikhailova N, Ovchinnikova G, Hooper SD, Lykidis A, Kyrpides NC. GenePRIMP: a gene prediction improvement pipeline for prokaryotic genomes. Nat Methods 2010; 7:455-457. PubMed doi:10.1038/nmeth.1457

37. Markowitz VM, Ivanova NN, Chen IMA, Chu K, Kyrpides NC. IMG ER: a system for microbial genome annotation expert review and curation. Bioinformatics 2009; 25:2271-2278. PubMed doi:10.1093/bioinformatics/btp393 